\title{
Genes for transport and metabolism of spermidine in Ruegeria pomeroyi DSS-3 and other marine bacteria
}

\author{
Xiaozhen Mou ${ }^{1, *}$, Shulei Sun $^{2}$, Pratibha Rayapati ${ }^{2}$, Mary Ann Moran ${ }^{2}$ \\ ${ }^{1}$ Department of Biological Sciences, Kent State University, Kent, Ohio 44242, USA \\ ${ }^{2}$ Department of Marine Sciences, University of Georgia, Athens, Georgia 30602, USA
}

\begin{abstract}
Spermidine, putrescine, and other polyamines are sources of labile carbon and nitrogen in marine environments, yet a thorough analysis of the functional genes encoding their transport and metabolism by marine bacteria has not been conducted. To begin this endeavor, we first identified genes that mediate spermidine processing in the model marine bacterium Ruegeria pomeroyi and then surveyed their abundance in other cultured and uncultured marine bacteria. $R$. pomeroyi cells were grown on spermidine under continuous culture conditions. Microarray-based transcriptional profiling and reverse transcription-qPCR analysis were used to identify the operon responsible for spermidine transport. Homologs from 2 of 3 known pathways for bacterial polyamine degradation were also identified in the $R$. pomeroyi genome and shown to be upregulated by spermidine. In an analysis of genome sequences of 109 cultured marine bacteria, homologs to polyamine transport and degradation genes were found in $55 \%$ of surveyed genomes. Likewise, analysis of marine metagenomic data indicated that up to $32 \%$ of surface ocean bacterioplankton contain homologs for transport or degradation of polyamines. The degradation pathway genes puuB ( $\gamma$-glutamyl-putrescine oxidase) and spuC (putrescine aminotransferase), which are part of the spermidine degradation pathway in $R$. pomeroyi, emerged as suitable targets for molecular-based studies of polyamine processing by marine bacterial communities. The frequency of genes encoding transport and catabolism of spermidine and related polyamines suggests an important role for these compounds in carbon and nitrogen budgets of marine bacterioplankton.
\end{abstract}

KEY WORDS: Polyamine $\cdot$ Transcriptomic analysis $\cdot$ Microarray $\cdot$ Marine bacteria $\cdot$ Dissolved organic nitrogen

Resale or republication not permitted without written consent of the publisher

\section{INTRODUCTION}

Spermidine and other polyamines are aliphatic organic compounds with multiple amino groups. They are synthesized by organisms across all 3 domains of life, playing vital roles in diverse cellular processes including nucleic acid and protein biosynthesis (Tabor \& Tabor 1985, Higashibata et al. 2000, Kusano et al. 2007) and biosilica precipitation in diatom frustule formation (Kroger et al. 2000, Sumper \& Kroger 2004). In the cytoplasm of bacteria and marine algae, intracellular soluble polyamine concentrations reach $\mathrm{mM}$ levels
(Tyms 1989, Marian et al. 2000). As free constituents in seawater, however, they are only found at nM levels (Jorgensen et al. 1993, Lee \& Jorgensen 1995, Nishibori et al. 2001, 2003), partly due to their active turnover by marine bacteria (Höfle 1984, Lee \& Jorgensen 1995).

Compared to extensive studies on the concentration and fate of other dissolved organic nitrogen compounds in seawater, such as dissolved free amino acids (DFAA), investigations of bacterially-mediated polyamine transformations have been rare (Höfle 1984, Lee \& Jorgensen 1995). Yet recent metagenomic and meta- 
transcriptomic sequencing of marine microbial communities have recovered homologs of genes predicted to be involved in spermidine and putrescine transport and metabolism (Venter et al. 2004, Poretsky et al. 2005, Mou et al. 2008), in some cases showing evidence of differential distribution with ocean depth (DeLong et al. 2006). Furthermore, a recent Sargasso Sea study identified spermidine transporter proteins from SAR11 as an abundant component of the surface seawater metaproteome (Sowell et al. 2009). This sequence-based evidence suggests that polyamines are important substrates for heterotrophic microorganisms in the ocean, with implications for the cycling of both nitrogen and carbon.

In the present study, we focused on spermidine use by the marine Roseobacter member Ruegeria pomeroyi DSS-3. Up to now, systematic investigations of spermidine transport and catabolism have been restricted to a few model bacteria with clinical and medical implications, such as Escherichia coli K12 (Shaibe et al. 1985) and Pseudomonas aeruginosa PAO1 (Lu et al. 2002, Dasu et al. 2006). However, Alphaproteobacteria in the Roseobacter lineage are prevalent in marine surface waters, where they account for up to $20 \%$ of the total bacterioplankton in coastal areas and $10 \%$ in open oceans (Giovannoni \& Rappe 2000). The genome sequence of $R$. pomeroyi contains a number of genes that might be involved in spermidine transport (Moran et al. 2004) and metabolism.

Transcriptional profiling using whole genome microarray analysis (Bürgmann et al. 2007) and qPCR was used to identify key genes for spermidine processing in Ruegeria pomeroyi, and to provide insights into transporter specificity. A comprehensive bioinformatic survey of polyamine-related genes in marine bacterial genomes and metagenomes confirmed the numerical abundance of polyamine transport and degradation genes among marine bacterioplankton, and identified 2 candidate genes for monitoring of polyamine transformations in situ.

\section{MATERIALS AND METHODS}

Culture conditions. Ruegeria pomeroyi DSS-3 cells were grown in a modified marine basal medium (MBM; Gonzalez et al. 2003) containing spermidine or putrescine as the sole carbon and nitrogen source. Serine was also used as a substrate to provide comparative expression data for an amino acid. The concentration of the 3 compounds was normalized to $3 \mathrm{mM}$ carbon (i.e. $0.43 \mathrm{mM}$ spermidine, $0.75 \mathrm{mM}$ putrescine, or $1 \mathrm{mM}$ serine, Table 1). Cells were grown in $200 \mathrm{ml}$ chemostats at $30^{\circ} \mathrm{C}$, a dilution rate of $0.125 \mathrm{~h}^{-1}$, an airflow rate of $1 \mathrm{ml} \mathrm{min}{ }^{-1}$, and a stirring speed of $200 \mathrm{rpm}$. Chemostat cultures were maintained at a constant cell density of $\mathrm{OD}_{600}=0.2$ for at least 4 retention times prior to harvesting. To harvest the cells, the outflow pumps were set to $6 \mathrm{ml} \mathrm{min}^{-1}$, and $9 \mathrm{ml}$ of cells were directly collected in chilled tubes containing $1 \mathrm{ml}$ stop solution (5\% phenol, 95\% ethanol, $\mathrm{pH}=8$ ). Chemostat cultures were established in quadruplicate for all 3 compounds in order to provide 4 independent replicates of each treatment for microarray or qPCR analysis.

RNA extraction, purification, and amplification. Cells harvested from the chemostat cultures were immediately centrifuged at $5000 \times g(10 \mathrm{~min})$ at $4^{\circ} \mathrm{C}_{\text {; }}$ the cell pellets were then frozen at $-80^{\circ} \mathrm{C}$ or used immediately for RNA extraction. Total RNA extraction, mRNA purification, and mRNA amplification to aminoallyl labeled antisense RNA (aa-aRNA) were performed following protocols described previously (Bürgmann et al. 2007).

Microarray hybridization and processing. The Ruegeria pomeroyi DSS-3 whole genome microarray was designed on a CombiMatrix Custom Array platform (Bürgmann et al. 2007). Along with probes for quality control, each array contains 12000 probes that target 4161 out of 4348 identified genes in the R. pomeroyi genome (mostly 2 probes per gene; Bürgmann et al. 2007). The genes that were excluded from the array either had close homologs in the genome or did not meet probe design criteria with regard to hybridization temperature.

The aa-aRNA was fluorescently labeled and hybridized to the Ruegeria pomeroyi microarray as described previously (Bürgmann et al. 2007), except that a non-competitive hybridization scheme was used,

Table 1. Structure and C:N ratio of 5 polyamine compounds used in the present study and the amino acid serine

\begin{tabular}{|c|c|c|c|}
\hline Compound & Formula & $\mathrm{C}: \mathrm{N}$ & Chemical Structure \\
\hline Cadaverine & $\mathrm{C}_{5} \mathrm{H}_{14} \mathrm{~N}_{2}$ & 2.5 & \\
\hline Norspermidine & $\mathrm{C}_{6} \mathrm{H}_{17} \mathrm{~N}_{3}$ & 2 & \\
\hline Putrescine & $\mathrm{C}_{4} \mathrm{H}_{12} \mathrm{~N}_{2}$ & 2 & $\mathrm{H}_{2}$ \\
\hline Spermidine & $\mathrm{C}_{7} \mathrm{H}_{19} \mathrm{~N}_{3}$ & 2.33 & \\
\hline Spermine & $\mathrm{C}_{10} \mathrm{H}_{26} \mathrm{~N}_{4}$ & 2.5 & \\
\hline Serine & $\mathrm{C}_{3} \mathrm{H}_{7} \mathrm{NO}_{3}$ & 3 & $\mathrm{H}_{2}$ \\
\hline
\end{tabular}


i.e. aa-aRNA was labeled only with a single dye (AlexaFluor dye 647; Invitrogen). After hybridization, the microarrays were scanned with an Axon GenPix 4000B microarray scanner (Molecular Devices Corporation) at $5 \mu \mathrm{m}$ resolution. Images were acquired and analyzed using GenePix Pro 6.0 software (Molecular Devices Corporation). The detection limits (DL) were calculated based on reading of the 149 empty spots on the array using the equation: DL = average sum of medians $+2 \times(\mathrm{SD})$. Spots with intensity below the DL and those identified as bad, empty, and not meeting quality assurance were excluded from further analysis.

Background corrected expression data from each array were globally normalized by trimmed means ( $2 \%$ from each side) and $\log _{2}$ transformed prior to being imported into the Acuity 4.0 software (Molecular Devices Corporation). Analysis datasets were created using the conditions (signal-to-noise ratio $>3$; circularity $>80 ; \mathrm{F} 635 \%<2$; B635 CV < 50) to exclude probes with features close to background, saturated, with bad circularity, or with highly non-uniform intensities or background.

Gap statistics predicted that the optimal cluster size for the array data was 3. Self Organizing Maps (SOM) cluster analysis was then performed within Acuity using a $1 \times 3$ cluster matrix, the Euclidean squared similarity metric, and data centering (Fig. S1 available as supplementary material at www.int-res.com/ articles/suppl/a058p311_app.pdf). The 4 experimental replicates were averaged to calculate the fold change between the spermidine samples and serine controls. $t$-test parameters and false discovery rates (FDR) were calculated to determine the significance of observed differences. Upregulated genes were reported when gene probes in spermidine samples showed more than 2-fold increase in expression level than those in serine controls, with $t$-test $\mathrm{p}$ values $<0.05$, false discovery rate $<10 \%$, and membership in SOM probe clusters that showed increased expression in the spermidine relative to the serine samples. Downregulated genes were reported when genes showed an opposite but equivalent response. The remainder of the genes were designated as non-responding. Microarray data were deposited in the Gene Expression Omibus database (www.ncbi.nlm.nih.gov) under accession number GLP4067.

RT-qPCR. For microarray result verification and transporter preference analysis, 12 genes were analyzed by reverse transcription (RT)-qPCR using mRNA obtained independently from that used for microarray analysis. The genes included each of the polyaminebinding components of 6 complete polyamine ABC transporter systems and another 2 genes randomly selected from each of the upregulated, downregulated, and non-responding genes. Primer sets for each gene were designed using Geneious Pro software (Biomatters) and are listed in Table S1 available as supplementary material at www.int-res.com/articles/suppl/ a058p311_app.pdf. The designed annealing temperature for each primer set ranged from 59 to $61^{\circ} \mathrm{C}$. The practical annealing temperature for all primer sets was chosen by performing a gradient PCR assay (annealing temperature varied between 57 and $65^{\circ} \mathrm{C}$ ) using the genomic DNA of Ruegeria pomeroyi as template, and this converged at $60^{\circ} \mathrm{C}$. Both aa-aRNA and nonamplified mRNA extracts that had only been treated for rRNA removal (mRNA only kit; Invitrogen) were used as RT-qPCR templates. RNA samples were quantified by spectrophotometer and were reverse transcribed to cDNA with random hexamer primers at a concentration of $0.3 \mu \mathrm{g} \mathrm{ll}^{-1}$ using iScript (Bio-Rad) according to the manufacturer's instructions. Triplicate qPCR reactions were conducted in $25 \mu \mathrm{l}$ volumes on an iCycler IQ multicolor Real-Time PCR detection System (Bio-Rad). Each reaction contained $1 \mu \mathrm{l}$ of cDNA template or standard, forward and reverse primers at a final concentration of $10 \mathrm{pM}$ each, and $1 \times$ IQ SYBR Green Supermix (Bio-Rad). Control reactions omitting template or reverse transcriptase were included for each analysis. The qPCR program contained an initial denaturation step $\left(95^{\circ} \mathrm{C}, 5 \mathrm{~min}\right)$ and 45 amplification cycles, each consisting of a denaturation step $\left(95^{\circ} \mathrm{C}\right.$, $45 \mathrm{~s})$, annealing step $\left(60^{\circ} \mathrm{C}, 90 \mathrm{~s}\right)$, and a final melting curve analysis. Standards were obtained from a dilution series of PCR amplicons of RNase-treated $R$. pomeroyi genomic DNA over 6 orders of magnitude for each primer set and used for calculating fold changes between treatments. For transporter substrate preference analysis, an identical RT-qPCR procedure was performed except that only the gene sets for the 6 polyamine-binding protein genes were used and mRNA was obtained from both spermidine- and putrescine-grown chemostat cultures.

Phylogenetic analysis. The amino acid sequences of the putative polyamine substrate-binding proteins in Ruegeria pomeroyi were aligned based on Blosum62 using ClustalW with the MEGA4 program (Kumar et al. 2004) and checked manually. The resulting distance matrix was used for generating a phylogenetic tree using the minimum evolutionary, neighbor-joining, and UPGMA algorithms with 1000 bootstraps using MEGA4.

Polyamine growth survey. Ruegeria pomeroyi DSS3 cells were grown in MBM containing the following single polyamine compounds as sole carbon and nitrogen sources: spermidine, putrescine, cadaverine, norspermine, and spermine. Serine provided comparative data for an amino acid. The concentration of each compound was normalized to $3 \mathrm{mM}$ carbon (i.e. $0.43 \mathrm{mM}$ spermidine, $0.75 \mathrm{mM}$ putrescine, $0.6 \mathrm{mM}$ 
cadaverine, $0.5 \mathrm{mM}$ norspermidine, $0.3 \mathrm{mM}$ spermine, and $1 \mathrm{mM}$ serine; Table 1). Cells were grown in the dark at $30^{\circ} \mathrm{C}$ with shaking at $200 \mathrm{rpm}$. Biomass was measured as the optical density of cells at a wavelength of $600 \mathrm{~nm}\left(\mathrm{OD}_{00}\right)$ at regular intervals until the cells reached a stationary phase. Batch cultures were established in triplicate.

Bioinformatic analysis. Polyamine transport systems (gene designation: $p o t$ ) were putatively identified from sequenced marine bacterioplankton genomes in the Moore Microbial Genome sequencing database (data freeze date: 1 October 2008; https://moore.jcvi.org/ moore/) based on key word searches. A blastp assay was also performed using identified polyamine-binding proteins in the genome of Escherichia coli K12 ( $p o t D$ and $p o t F$ ), with an E value cutoff of $<10^{-20}$. The candidates from the 2 procedures were combined and then manually inspected for orthology to polyamine transporters and to ensure that each identified pot system contained consecutive genes that encoded at least 1 copy of each of the 4 components, which is required for a polyamine transporter to function. Polyamine degradation genes do not require consecutive gene systems to function. A reciprocal best hit methodology with an $E$ value cutoff of $<10^{-30}$ was used to identify orthologs to $p u u B$ and $s p u C$ in the sequenced marine bacterioplankton genomes in the Moore genome database.

Homologs to experimentally confirmed polyaminebinding proteins ( $p o t D$ and potF) were identified in the Global Ocean Survey (GOS) dataset using blastp with an E value cutoff of $<10^{-20}$. The 10 GOS hits at the boundary of the cutoff were blasted back to the NCBI Refseq database (www.ncbi.nlm.nih.gov/RefSeq/) and the Escherichia coli and Ruegeria pomeroyi genomes. If 2 or more hits were not to the correct functional category, the E value cutoff was decreased by 5 orders of magnitude for a subsequent blast. The same procedure was adopted for the identification of homologs to $R$. pomeroyi SPO3465 (predicted as puuB) and SPO3473 (predicted as spuC) in the GOS dataset. The final cutoff for all analyses converged on an E value of $<10^{-30}$. The acetylornithine aminotransferase gene ( $\arg D$ ) homolog in $R$. pomeroyi (SPO0962) has a related but distinct function to spuC. The blast hits that had a higher bit score for $\arg D$ than $s p u C$ in blastp analyses were removed from the final list of spuC homologs. Paired read sequences in GOS data were only counted once.

Homologs to polyamine synthesis genes in Ruegeria pomeroyi were obtained by blasting known genes from E. coli K12 (Blattner et al. 1997, http://ecoli.naist.jp/ GB6/search.jsp) and Pseudomonas aeruginosa PAO1 (Stover et al. 2000, www. pseudomonas.com) to the $R$. pomeroyi genome sequence using blastp through the RoseoBase website (www.roseobase.org). Gene orthologs were reported when the reciprocal best hit E value was $<10^{-30}$.

\section{RESULTS}

\section{Microarray analysis and quality control}

Ruegeria pomeroyi cells were grown on spermidine or serine under steady-state conditions in a chemostat with fixed cell growth rates, temperature, $\mathrm{pH}$, and air flow. This effort minimized gene expression artifacts due to factors other than substrate differences. Of the 4161 genes that were arrayed, significant increases in mRNA levels were found for 92 genes in spermidine treatments relative to serine treatments (i.e. $\geq 2$-fold higher normalized fluorescence; $t$-test, $\mathrm{p}<0.05$; Table 2); these were designated as upregulated genes (Fig. 1). About one-third of the upregulated genes

Table 2. Ruegeria pomeroyi. Upregulated genes hypothesized to be involved in spermidine transport and degradation by $R$. pomeroyi. Complete lists of up- and downregulated genes are provided in Tables S2 \& S3 available as supplementary material at www.int-res.com/articles/suppl/a058p311_app.pdf

\begin{tabular}{|lccl|}
\hline Gene locus tag & COG & Gene name & Annotation \\
\hline SPO1300 & COG0174 & & Glutamine synthetase family protein \\
SPO1301 & COG0518 & & $\begin{array}{l}\text { Glutamine amidotransferase class I } \\
\text { Glutamine synthetase family protein }\end{array}$ \\
SPO1302 & COG0174 & & Glutamate/aspartate ABC transporter, permease protein \\
SPO3465 & COG0765 & gltJ & Conserved hypothetical protein \\
SPO3466 & COG1177 & potI & Putrescine ABC transporter, permease protein \\
SPO3467 & COG1176 & potH & Putrescine ABC transporter, permease protein \\
SPO3468 & COG3842 & potG & Putrescine ABC transporter, ATP-binding protein \\
SPO3469 & COG0687 & potF & Putrescine ABC transporter, periplasmic substrate-binding protein \\
SPO3471 & COG0161 & & Aminotransferase class III \\
SPOA0273 & & & DNA-binding protein, putative \\
\hline
\end{tabular}




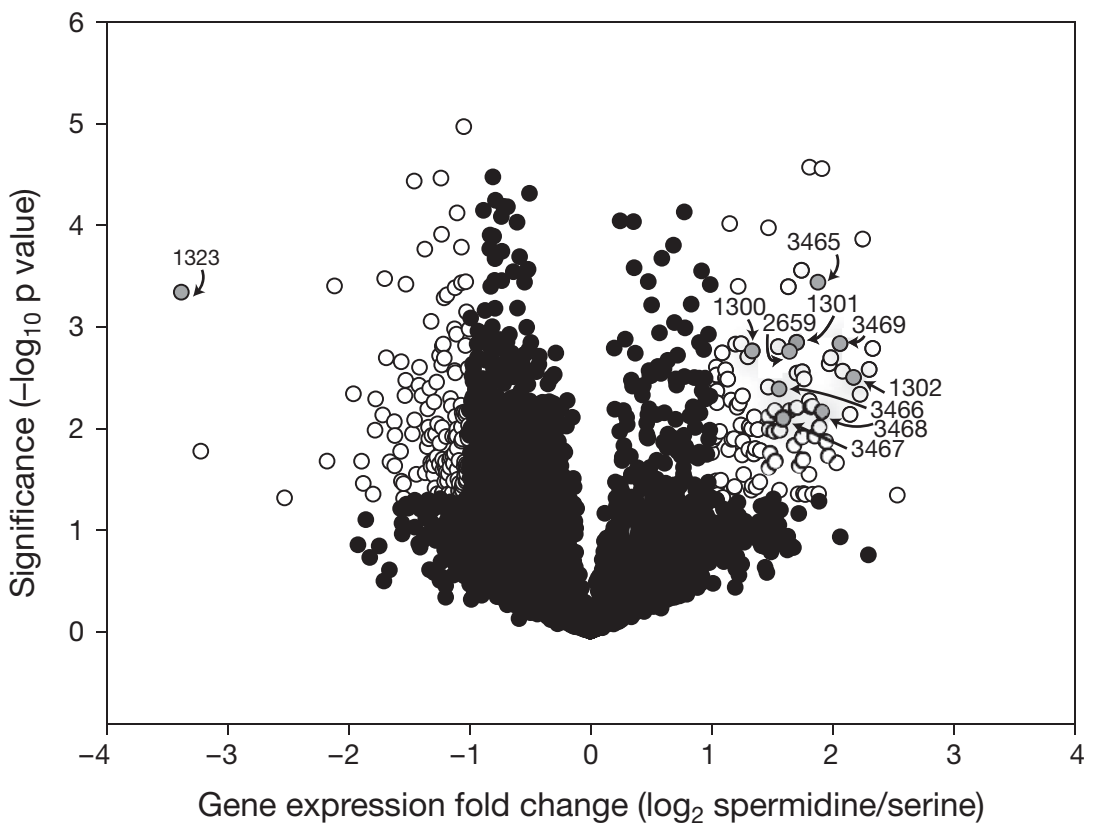

Fig. 1. Ruegeria pomeroyi. Transcriptional response of genes grown on spermidine relative to serine shown on a volcano plot. Up- or downregulated genes $(\geq 2$ fold expression change; $\mathrm{p}<0.05$ ) in spermidine treatments relative to the serine treatments are illustrated as open or gray circles, with locus tag coding of the gray circles the same as in Tables 2, S2, \& S3. Genes showing no significant transcriptional changes are illustrated as black circles
(29 genes) had sequences too divergent to allow annotation of even general function (Table S2 provided as supplementary material at www.int-res.com/articles/ suppl/a058p311_app.pdf). Most of the remainder represented functions involved in substrate transport (12 genes), nitrogen metabolism (12), carbon metabolism (10), and protein regulation (10). As expected, the L-serine ammonia-lyase gene ( $\operatorname{sda} A)$, a gene for serine degradation, was downregulated in the spermidine treatment relative to serine $(t$-test, $\mathrm{p}<$ $0.05)$, as were other serine metabolismrelated genes (Table S3). RT-qPCR using original mRNA extracts and amino-allyl labeled antisense RNA (aa-aRNA, the same form as the microarray template) agreed well with each other and both agreed with the microarray data (Fig. 2).

\section{Transport genes upregulated by spermidine}

In bacteria, exogenous polyamines are thought to be mainly transported by ATPbinding cassette transport systems (ABCtype transporters; Tabor \& Tabor 1985). Each polyamine transport system (gene designation: pot) typically consists of 4 indispensable components that are encoded by contiguous genes, i.e. 1 periplasmic substrate- binding protein, 2 hydrophobic integral membrane proteins (permeases), and 1 hydrophilic peripheral membrane
ATP-binding protein. The genes of the substratebinding proteins are the most divergent, while the genes of the ATP-binding proteins are the most conserved (Tam \& Saier 1993, Saurin \& Dassa 1994).

In the Ruegeria pomeroyi genome, 6 complete sets of 4-component pot systems have been predicted based on sequence homology (Moran et al. 2004). Only 1 set, SPO3466-SPO3469, was upregulated by exogenous spermidine in the microarray analysis (Table 2) and,

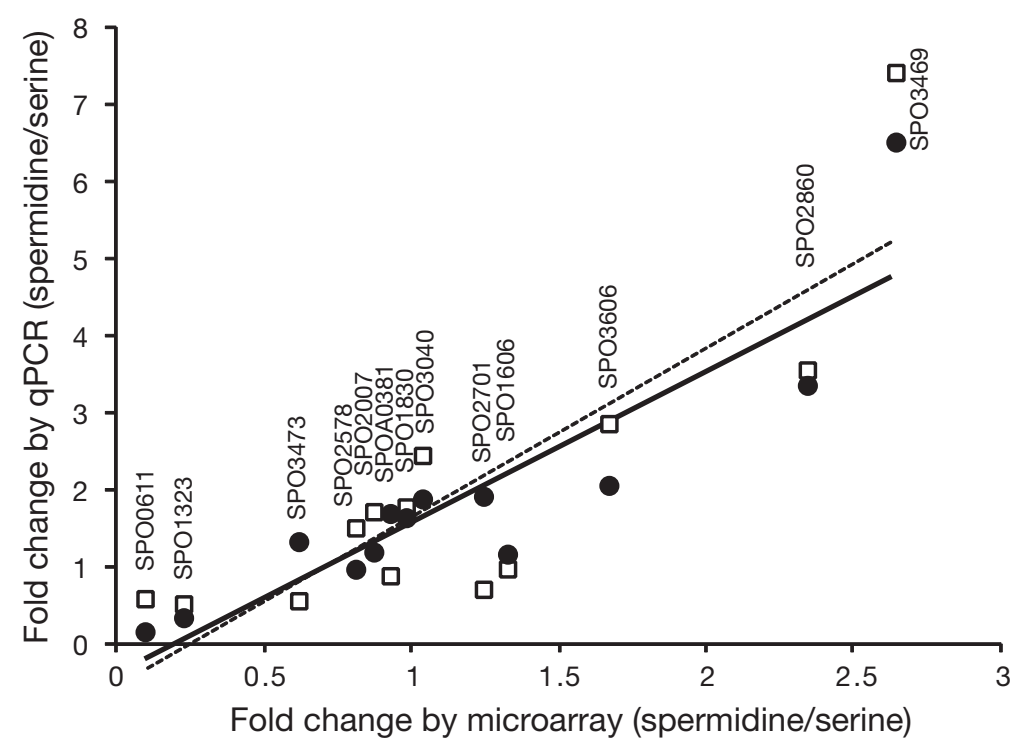

Fig. 2. Ruegeria pomeroyi. Validation of microarray data shown by correlations with RT-qPCR-based analysis of expression level changes relative to serine. Closed circles, solid trend line: mRNA; open squares, dashed trend line: amino-allyl labeled antisense RNA. Gene locus tags are labeled adjacent to corresponding data points (see Tables 2, S2 \& S3) 
unexpectedly, this system has been annotated as a putrescine-specific transport system with gene designations potFGHI. Comparing the amino acid sequence of the substrate-binding protein of the upregulated transporter system, SPO3469, to experimentally confirmed substrate-binding proteins in other bacteria, we found high similarity to both putrescine and spermidine-binding proteins. SPO3469 was 50\% identical in amino acid sequence to potF (experimentally shown to be specific for putrescine binding in Escherichia coli), $30 \%$ identical to potD (experimentally shown to bind both spermidine and putresine in E. coli, but with a preference for the former; Igarashi \& Kashiwagi 1999), and $51 \%$ and $47 \%$ identical to spuD and $s p u E$, the 2 preferential spermidine-binding proteins in a 5-component pot system (SpuDEFGH) of Pseudomonas aeruginosa (Lu et al. 2002).

Amino acid sequence alignments were used to assess residue conservation and phylogenetic relatedness of the binding protein upregulated in response to spermidine (SPO3469), the other 5 putative pot system binding proteins in Ruegeria pomeroyi, and the experimentally confirmed spermidine- and putrescinebinding proteins in Escherichia coli and Pseudomonas aeruginosa. SPO3469 and another putative pot binding protein gene from $R$. pomeroyi (SPO1606) consistently clustered with potD and potF from E. coli and spuD and spuE from $P$. aeruginosa (Fig. 3). However, SPO3469 is the only polyamine-binding protein in the $R$. pomeroyi genome that is conserved for all 3 amino acid residues experimentally demonstrated to be the most critical to spermidine binding by potD in E. coli (Igarashi \& Kashiwagi 1999; Fig. S2).

\section{Substrate preference of Ruegeria pomeroyi pot systems}

The high sequence divergence of polyamine-binding protein genes among the 6 pot systems in the Ruegeria pomeroyi genome (averaging only 19\% sequence identity; Fig. 3) suggests differing substrate preferences. This idea is consistent with the high redundancy of pot transporters (6 sets) in the $R$. pomeroyi genome, as well as the apparent expression of only 1 of these in response to growth on spermidine. To obtain additional information on transporter specificity, the relative transcriptional response of the 6 polyaminebinding genes was compared by RT-qPCR

$$
\vdash_{0.1}
$$

for chemostat-grown cells with spermidine or putrescine as the substrate. SPO3469 had 6.5-fold increased transcription during growth on spermidine, in accordance with the microarray data. SPO3473 and SPO1606 showed no upregulation in response to spermidine, but had 3.2- and 4.5-fold increased transcription during growth on putrescine (Table 3). SPO2007, SPO2701, and SPOA0381, the putative polyaminebinding protein genes in 3 other pot systems in the $R$. pomeroyi genome (Fig. 3), were not significantly upregulated by either spermidine or putrescine, and likely transport other polyamines or related compounds. Batch culture assays indicated that at least 3 additional polyamines (cadaverine, norspermidine, and spermine; Table 1) could be transported and metabolized by $R$. pomeroyi (Fig. S3 available as supplementary material at www.int-res.com/articles/ suppl/a058p311_app.pdf).

\section{Catabolic genes upregulated by spermidine}

Three pathways have been hypothesized previously to mediate bacterial spermidine degradation (Fig. 4).

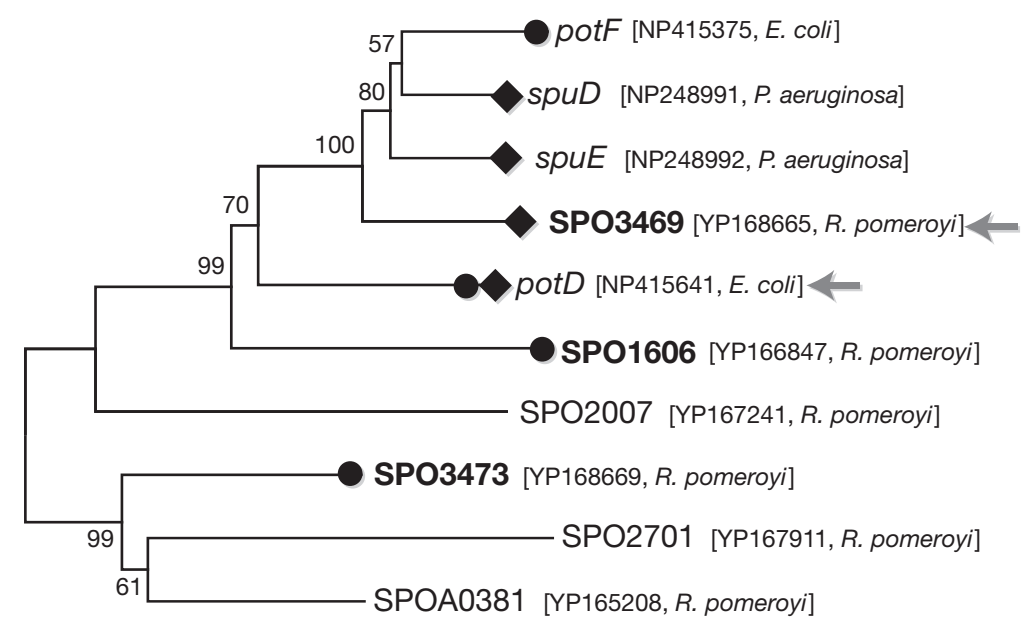

Fig. 3. Ruegeria pomeroyi. Phylogenetic tree based on amino acid sequences of polyamine-binding proteins in experimentally confirmed pot systems and in 6 putative pot systems in the genome of $R$. pomeroyi. The tree was constructed using minimum evolution with 1000 bootstrap replications, and bootstrap values $>50 \%$ are indicated at the branch nodes. Scale bar indicates the amount of genetic change in terms of the number of amino acid substitutions per site. GenBank accession numbers and source organisms are shown in brackets. Bold font indicates $R$. pomeroyi spermidine and putrescine transporter genes identified in the present study. Black circles and diamonds indicate binding proteins shown experimentally to transport putrescine and spermidine, respectively. Gray arrows indicate binding proteins with 3 conserved amino acids hypothesized to be critical for spermidine binding (Igarashi \& Kashiwagi 1999). Other tree-building algorithms (UPGMA, neighbor joining) resulted in nearly identical branching patterns 
Table 3. Ruegeria pomeroyi. Average fold changes in abundance of transcripts for polyamine-binding protein genes of $R$. pomeroyi based on RT-qPCR of chemostat-grown cells in polyamine treatments relative to serine treatments. Each fold-change result was an average of 9 sets of data ( 3 RT-qPCR technical replicates $\times 3$ chemostat samples)

\begin{tabular}{|lcccccc|}
\hline Samples & SPO1606 & SPO2007 & SPO2701 & SPO3469 & SPO3473 & SPOA0381 \\
\hline Putrescine mRNA $^{\mathrm{a}}$ & 3.2 & 0.9 & 1.8 & 1.4 & 4.5 & 1.1 \\
Spermidine mRNA $^{\mathrm{a}}$ & 1.1 & 1.2 & 1.9 & 6.5 & 1.3 & 1.7 \\
Spermidine aa-aRNA $^{\mathrm{b}}$ & 1.1 & 1.7 & 0.7 & 7.4 & 0.6 & 0.9 \\
${ }^{\text {amRNA used as template; }}{ }^{\mathrm{b}}$ amino-allyl-labeled antisense RNA used as template & & & \\
\hline
\end{tabular}

Two of them channel spermidine through putrescine degradation pathways, and these are further divided into the transamination (Lu et al. 2002) and $\gamma$-glutamylation routes (Kurihara et al. 2005). The third pathway involves oxidative cleavage of spermidine into 4aminobutyraldehyde and 1,3-diaminopropane before further degradation to cellular intermediates (Dasu et al. 2006). All 3 routes produce intermediates for the tricarboxylic acid (TCA) cycle and other cellular processes. The microarray data suggested that 2 of these pathways are operational in Ruegeria pomeroyi. All key genes of the transamination and $\gamma$-glutamylation routes have putative homologs in the genome of $R$. pomeroyi, and as detailed below, many were upregulated during growth on spermidine (Table 2, Fig. 4).

Upregulated genes SPO3465 and SPO3471 are located on either side of the upregulated pot transport system SPO3466SPO3469 (Fig. 5). SPO3465 shares 30\% identity with the $\gamma$-glutamyl-putrescine oxidase gene of Escherichia coli (puuB; Kurihara et al. 2005) in the $\gamma$-glutamylation route of putrescine degradation (Fig. 3). SPO3471 shares $60 \%$ identity with the putrescine aminotransferase gene (spuC), which catalyzes the removal of 1 of the 2 amino groups from putrescine (Lu et al. 2002; Fig. 4). The adjacent gene SPO3470 had a higher expression during growth on spermidine compared to serine (although it did not meet the significance criteria) and is annotated as a GntR-type transcriptional regulator. The 7 consecutive genes SPO3465- SPO3471 together appear to form a cluster that functions in both spermidine uptake and degradation (Fig. 5).

The upregulated genes SPO1300 and SPO1302 are both similar to the $\gamma$-glutamyl-putrescine synthase gene puuA (both 35\% identical), which catalyzes the first step of the $\gamma$-glutamylation pathway in putrescine degradation (Kurihara et al. 2005; Fig. 4). This observed duplication of
puuA homologs is consistent with the Pseudomonas aeruginosa genome $(s p u B$ and spuI, both of which have been shown experimentally to function in spermidine degradation; Lu et al. 2002). SPO1301 is annotated as a glutamine amidotransferase (GATase), which mediates the biosynthesis of a variety of organic nitrogen compounds including nucleotides. Putative GATases in Escherichia coli (designated puuD) and $P$. aeruginosa (designated spuA) have been shown to be involved in spermidine degradation ( $\mathrm{Lu}$ et al. 2002, Kurihara et al. 2005), but both have low identities to SPO1301 ( 15\% identical in each case). While SPO1300-SPO1302 are located distantly from

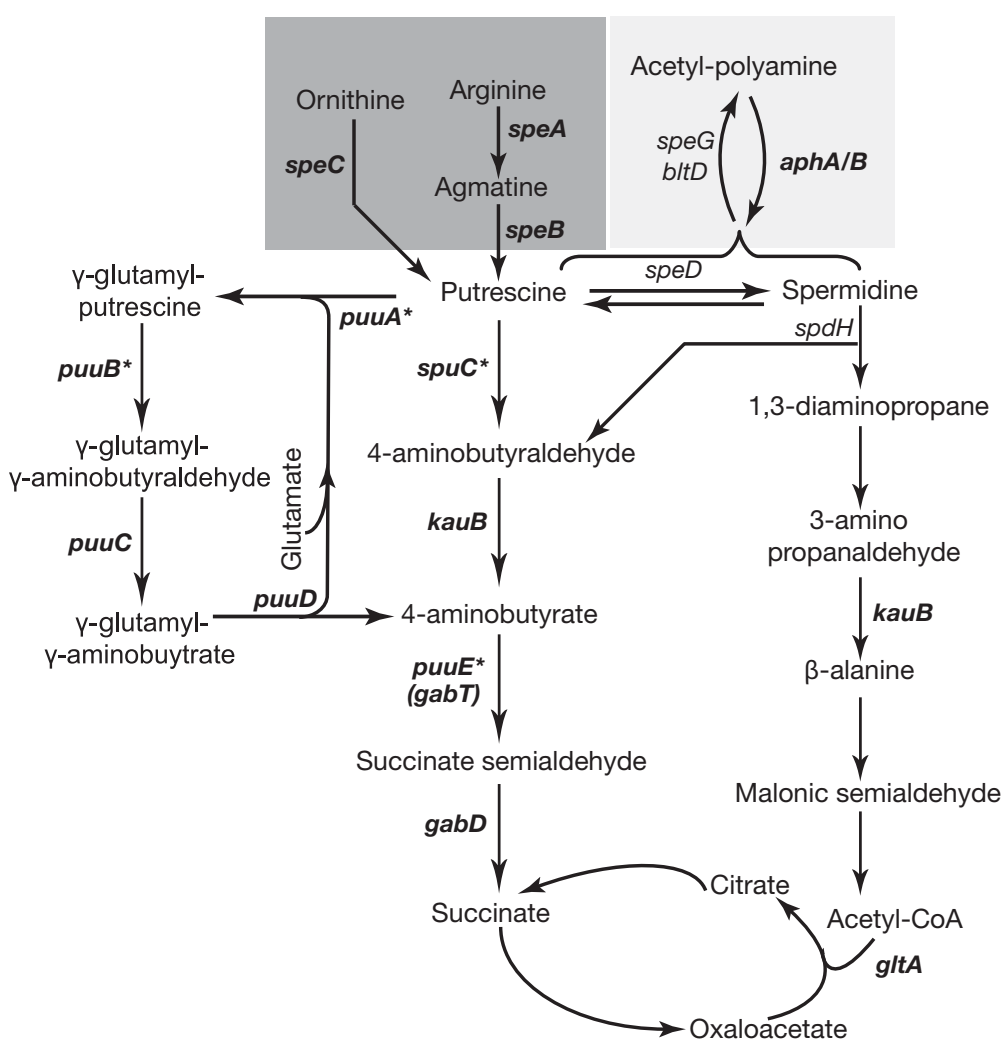

Fig. 4. Hypothesized spermidine degradation pathways in bacteria. Gene names with normal font: no homolog in Ruegeria pomeroyi; bold font: homolog in $R$. pomeroyi; bold font and asterisk: homolog upregulated by spermidine in $R$. pomeroyi; arrow with no gene label: gene has not yet been identified (modified after Dasu et al. 2006, Chou et al. 2008) 


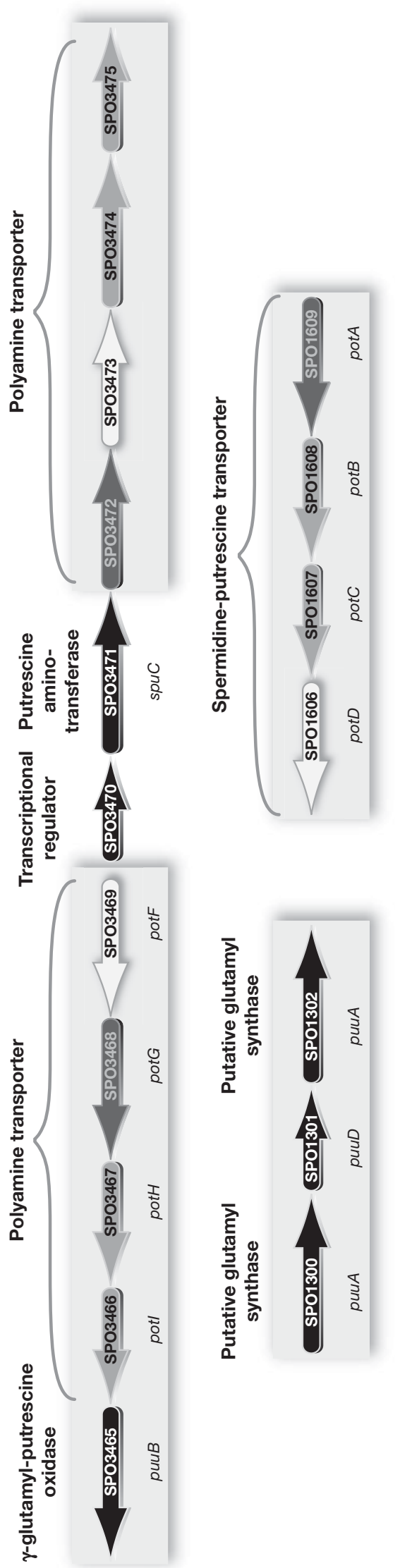

SPO3465-SPO3471 in the genome of Ruegeria pomeroyi (Fig. 5), orthologs have been found in a single cluster in other genomes (Lu et al. 2002, Kurihara et al. 2005). Upregulation of SPO2659, a putative glutamate transport gene $(g l t J)$, might indicate a higher demand for glutamate in response to increased activity of the $\gamma$-glutamylation degradation route.

Increased expression of many homologs of genes with demonstrated function in putrescine degradation indicates that spermidine is converted to putrescine in Ruegeria pomeroyi (Table 2, Fig. 4), as has been hypothesized in a Pseudomonas strain (Padmanabhan $\&$ Kim 1965). We found no homologs for the third pathway for spermidine degradation involving direct oxidative cleavage that has been demonstrated in P. aeruginosa (Dasu et al. 2006; Fig. 4). Overall, the microarray and qPCR data indicate that $R$. pomeroyi transports exogenous spermidine into the cell by a dedicated ABC-type transporter, degrades it to putrescine, and then ultimately to the TCA cycle intermediate succinate. Ammonia, alanine, and glutamate are also generated along the degradation pathway (Fig. 4), satisfying both carbon and nitrogen demands of the cell.

\section{Polyamine genes in marine bacterial genomes and metagenomes}

A bioinformatic survey of 109 marine heterotrophic bacterioplankton genomes identified a total of 196 complete polyamine transport systems in 74 genomes from many major bacterioplankton groups (Table 4). Complete transporter gene sets are found in all Actinobacteria, Rhizobiales, Roseobacter, SAR11, Oceanospirillales, and Vibrionales genomes $(2,4,2,2$, and 10 genomes, respectively). Groups with few or no transporters include Bacteriodetes and Planctomyces (15 and 2 genomes, respectively). Multiple pot systems are often found in the same genome. For example, roseobacters average $>4$ pot systems per sequenced genome. Some bacterioplankton, such as the 2 SAR11 representatives, are equipped with only a single pot system. Because polyamine-binding proteins may not cluster according to substrate specificities in phylogenetic analyses (Fig. 3), the substrates of these marine bacterioplankton pot transporters could not be identified further.

pot systems were also found with high frequency in the GOS metagenomic dataset, based on blastp queries using experimentally-confirmed polyaminebinding protein genes (Venter et al. 2004, Rusch et al. 2007; Table 5). Ratios of putative pot binding proteins to universal single-copy genes (Howard et al. 2008) indicate that as many as $32 \%$ of surface marine bacte- 
Table 4. Frequency of genes for polyamine transport ( $p o t$ ) and degradation ( $p u u B$ and $s p u C$ ) in 109 sequenced marine bacterioplankton genomes. Table entries show the number of multi-gene systems (pot) or single genes (puuB and spuC) per genome. Only taxa with positive results are listed. The notation in parenthesis shows the number of positive genomes/ total number of genomes surveyed for each group.

\begin{tabular}{|c|c|c|c|}
\hline \multirow{2}{*}{$\frac{\text { Bacterioplankton taxa }}{\text { Alphaproteobacteria }}$} & \multicolumn{2}{|c|}{ рuuB } & spuC \\
\hline & & & \\
\hline \multicolumn{4}{|l|}{ Roseobacter (23/23) } \\
\hline Alpha proteobacterium HTCC2255 & 3 & 1 & 1 \\
\hline Loktanella vestfoldensis SKA53 & 1 & 1 & \\
\hline Oceanicola batsensis HTCC2597 & 3 & 1 & 1 \\
\hline Oceanicola granulosus HTCC2516 & 2 & 1 & \\
\hline Oceanibulbus indolifex HEL-45 & 3 & 1 & 1 \\
\hline Octadecabacter antarcticus 307 & 4 & 1 & \\
\hline Phaeobacter gallaeciensis 2.10 & 3 & 1 & 1 \\
\hline Phaeobacter gallaeciensis BS107 & 2 & 1 & 1 \\
\hline Rhodobacterales bacterium HTCC2150 & 2 & 1 & 1 \\
\hline Rhodobacterales bacterium HTCC2654 & 2 & 1 & 1 \\
\hline Roseobacter litoralis Och 149 & 6 & 1 & 1 \\
\hline Roseobacter sp. AzwK-3b & 3 & 1 & 1 \\
\hline Roseobacter sp. CCS2 & 2 & 1 & 1 \\
\hline Roseobacter sp. MED193 & 2 & 1 & 1 \\
\hline Roseobacter sp. SK209-2-6 & 4 & 1 & 1 \\
\hline Roseovarius nubinhibens ISM & 7 & 1 & 1 \\
\hline Roseovarius sp. 217 & 8 & 1 & 1 \\
\hline Roseovarius sp. HTCC2601 & 3 & 1 & 1 \\
\hline Roseovarius sp. TM1035 & 5 & & 1 \\
\hline Ruegeria pomeroyi DSS-3 & 6 & 1 & 1 \\
\hline Sagittula stellata E-37 & 6 & 1 & 1 \\
\hline Sulfitobacter sp. EE-36 & 2 & 1 & 1 \\
\hline Sulfitobacter sp. NAS-14.1 & 2 & 1 & 1 \\
\hline \multicolumn{4}{|l|}{ Other Rhodobacterales (2/3) } \\
\hline Stappia aggregata IAM 12614 & 5 & 1 & 1 \\
\hline Stappia alexandrii DFL-11 & 2 & 1 & 1 \\
\hline \multicolumn{4}{|l|}{ SAR11 (2/2) } \\
\hline Pelagibacter ubique HTCC1002 & 1 & 1 & \\
\hline Pelagibacter sp. HTCC7211 & 1 & 1 & \\
\hline \multicolumn{4}{|l|}{ Rhizobiales (4/4) } \\
\hline Aurantimonas sp. SI85-9A1 & 1 & 1 & \\
\hline Fulvimarina pelagi HTCC2506 & 1 & 1 & \\
\hline Hoeflea phototrophica DFL-43 & 2 & 1 & 1 \\
\hline Nitrobacter sp. Nb-311A & 1 & & \\
\hline \multicolumn{4}{|l|}{ Other alpha (1/7) } \\
\hline Alpha proteobacterium BAL199 & 4 & 1 & 1 \\
\hline \multicolumn{4}{|l|}{ Gammaproteobacteria } \\
\hline \multicolumn{4}{|l|}{ Alteromonadales (6/9) } \\
\hline Alteromonas macleodii Deep ecotype & & 1 & \\
\hline Marinobacter algicola DG893 & 2 & & \\
\hline Marinobacter sp. ELB17 & 4 & 1 & \\
\hline Moritella sp. PE36 & 1 & & \\
\hline Pseudoalteromonas tunicata D2 & 1 & 1 & \\
\hline Shewanella benthica KT99 & 1 & & 1 \\
\hline \multicolumn{4}{|l|}{ Oceanospirillales (3/3) } \\
\hline Marinomonas sp. MED121 & 3 & 1 & 1 \\
\hline Oceanobacter sp. RED65 & 2 & 1 & 1 \\
\hline Oceanospirillum sp. MED92 & 2 & 1 & 1 \\
\hline \multicolumn{4}{|l|}{ Vibrionales (10/10) } \\
\hline Photobacterium profundum 3ТCK & 2 & 1 & \\
\hline Photobacterium sp. SKA34 & 3 & 1 & \\
\hline Vibrio alginolyticus 12G01 & 4 & 1 & \\
\hline Vibrio angustum S14 & 4 & 1 & \\
\hline Vibrio campbellii AND4 & 2 & & \\
\hline
\end{tabular}

Table 4 (continued)

\begin{tabular}{|lccc|}
\hline Bacterioplankton taxa & pot & puuB & spuC \\
\hline Vibrio fischeri MJ11 & 2 & & \\
Vibrio shilonii AK1 & 4 & 1 & 1 \\
Vibrio sp. MED222 & 2 & 1 & \\
Vibrio splendidus 12B01 & 2 & 1 & \\
Vibrionales bacterium SWAT-3 & 2 & 1 & \\
Other gamma (3/6) & & & \\
Congregibacter litoralis KT71 & & 1 & 1 \\
Marine gamma HTCC2080 & 1 & & 1 \\
Reinekea sp. MED297 & & & \\
Actinobacteria (2/2) & 1 & & 1 \\
Janibacter sp. HTCC2649 & 2 & & 1 \\
Marine actinobacterium PHSC20C1 & & & \\
Firmicutes (3/4) & 1 & & \\
Bacillus sp. B14905 & 1 & & \\
Bacillus sp. NRRL B-14911 & 1 & & \\
Carnobacterium sp. AT7 & & & \\
Bacteroidetes & & & 1 \\
Flavobacteria (1/12) & & & \\
Psychroflexus torquis ATCC 700755 & & \\
Other bacteroidetes (1/3) 3134 & & & \\
Microscilla marina ATCC 23134 & & & \\
Cyanobacteria (2/10) & & \\
Lyngbya aestuarii CCY9616 & 2 & & \\
Prochlorococcus marinus MIT 9211 & & 1 & \\
Planctomyces (0/2) & & \\
Other (3/10) & & \\
Caminibacter mediatlanticus TB-2 & 1 & \\
Marinitoga piezophila KA3 & & \\
Plesiocystis pacifica SIR-1 & & & \\
\hline
\end{tabular}

rioplankton cells could contain a pot system. This number is an overestimate of per-cell frequency if multiple pot systems are present in some bacterioplankton genomes, as is the case for cultured roseobacters (Table 4 ), or if 1 pot system contains $>1$ polyaminebinding protein, as is the case for Pseudomonas aeruginosa (Lu et al. 2002). This frequency is lower than that found for pot systems in cultured marine bacterioplankton $(68 \%$; Table 4$)$.

\section{In situ probes for polyamine degradation}

Homologs to Ruegeria pomeroyi spermidine degradation genes SPO3465 (puuB) and SPO3473 (spuC) (putrescine transamination and $\gamma$-glutamylation pathways, respectively) were found with high frequency in sequenced marine bacterioplankton genomes and the GOS metagenomic dataset (Tables 4 \& 5). Of the 109 genomes examined, 47 puuB and 36 spuC orthologs were identified (reciprocal best hits in blastp analyses, Table 4). These putative orthologs are distributed widely among major marine bacterial taxa, and are particularly prevalent in the Roseobacter lineage. The 2 representative bacteria in the SAR11 clade each had a single ho- 
Table 5. Frequency of genes for polyamine transport (substrate-binding proteins potD and potF) and degradation (puuB and spuC) in the Global Ocean Sampling (GOS) metagenomic data, expressed as homolog number (and \% of cells \pm SD), assuming no more than one gene copy per cell

\begin{tabular}{|c|c|c|c|c|}
\hline GOS sites $^{\mathrm{a}}$ & Genomes sampled $^{\mathrm{b}}$ & $\operatorname{potD} / F$ & puuB & spuC \\
\hline Coastal (n = 19) & 1222 & $409(32 \pm 12 \%)$ & $108(10 \pm 7 \%)$ & $189(17 \pm 11 \%)$ \\
\hline Open ocean $(n=13)$ & 1334 & $500(32 \pm 11 \%)$ & $183(11 \pm 7 \%)$ & $340(24 \pm 16 \%)$ \\
\hline Hypersaline $(\mathrm{n}=1)$ & 298 & $44(15 \%)$ & $17(6 \%)$ & $40(13 \%)$ \\
\hline Estuary and Other $(\mathrm{n}=10)$ & 962 & $206(21 \pm 14 \%)$ & $39(5 \pm 7 \%)$ & $66(10 \pm 11 \%)$ \\
\hline
\end{tabular}

molog of these genes (Table 4). Assuming a single copy per cell, puuB and spuC homologs are present in $10 \%$ and $17 \%$ of bacterioplankton cells in coastal metagenomic libraries, and $11 \%$ and $24 \%$ in open ocean libraries (Table 5; frequencies are not significantly different between coastal and open ocean GOS sites; $t$-test, $p$ $>0.05$ ). For most marine bacterial genomes, puuB and spuC co-occur with pot system genes for transport of exogenous polyamines into the cell (Table 4).

\section{DISCUSSION}

Intracellular polyamines are found in virtually all living organisms at cellular concentrations in the micromolar range. While this source of organic carbon and nitrogen should be valuable to marine heterotrophic bacteria, whose growth in seawater is often limited by carbon or nitrogen, bacterially-mediated polyamine transformation has been studied in only a few cases. Results generated from the early studies are somewhat contradictory in terms of the fate of the carbon in exogenous polyamines. In one study, exogenous ${ }^{14} \mathrm{C}$-putrescine was preferentially respired to $\mathrm{CO}_{2}(\sim 85 \%)$ rather than incorporated in biomass ( $6 \%)$ by assemblages of marine bacterioplankton (Höfle 1984). In another, the same polyamine appeared to be largely incorporated into cell biomass by bacterial assemblages in a coastal salt pond (40 to $75 \%$ in an oxic zone; 60 to $100 \%$ in an anoxic zone; Lee \& Jorgensen 1995). In Ruegeria pomeroyi, spermidine is degraded to intermediates that feed into the tricarboxylic acid cycle, from which both energy generation and biosynthesis are possible, and growth rates and biomass yields are similar to those on the amino acid serine. The high concentrations used in the present study (up to 1 $\mathrm{mM}$ ) confirm that polyamines are not toxic to $R$. pomeroyi, as was shown by Höfle (1984) for a marine bacterial assemblage. Whether the fate of polyamine-derived carbon and nitrogen (i.e. incorporation versus regeneration) is affected by the concentration of polyamines and the supply of other labile DOM is yet to be determined.
The substrate specificity of polyamine transporters in marine bacterial genomes and metagenomes is hard to decipher at present. Whole-genome microarray and RT-qPCR data indicate that Ruegeria pomeroyi transporter systems may have narrow substrate specificities. This is further supported by the low identity $(\sim 19 \%)$ of amino acid sequences among predicted polyamine-binding proteins (Fig. 3). The clustering pattern of binding proteins cautions against the use of sequence identity alone as an indicator of substrate specificity among the R. pomeroyi, Escherichia coli, and Pseudomonas aeruginosa binding proteins analyzed here (Fig. 3). Because of their high sequence divergence, polyamine-binding proteins are not good targets for the design of general qPCR primer sets for environmental studies; even orthologs in genomes of 2 closely related strains for which 16S rRNA genes are $>96 \%$ identical (e.g. R. pomeroyi DSS-3 and Ruegeria sp. TM1040) presented a significant challenge for primer design. Our analyses suggest instead that the well-conserved degradation genes puuB and spuC are more robust targets for in situ probing of polyamine processing by natural bacterial communities.

Studies of ecologically-relevant model bacteria that are designed and interpreted in the context of metagenomic data from natural bacterial communities can significantly enhance our understanding of biogeochemical processes. Despite recognition of the importance of spermidine and other polyamines in seawater over 2 decades ago, limited information had been generated on the biological processing of these compounds. This study provides new details on the function and distribution of genes for transport and metabolism of spermidine in a model marine bacterium. Moreover, it shows a high frequency and wide taxonomic distribution of homologs to polyamine transport genes (pot) and degradation genes (puuB and spuC) in marine bacterioplankton communities (Tables 4 \& 5), arguing for increased attention to the role of polyamines in the marine carbon and nitrogen cycles. Functional characterization of these and other ecologically relevant 
bacterial genes is a critical task for postgenomic environmental microbiology.

Acknowledgements. We thank J. Henriksen and W. Ye for assistance with the chemostats; R. Poretsky, S. Gifford, and J. Rinta-Kanto for helpful discussions on microarray data analysis; C. English for graphic support; and G. Burnham and C. Sloan for technical assistance. This research was supported by a National Science Foundation Grant to the Sapelo Island Microbial Observatory (MCB0702125) and the Gordon and Betty Moore Foundation.

\section{LITERATURE CITED}

Blattner FR, Plunkett G, Bloch CA, Perna NT and others (1997) The complete genome sequence of Escherichia coli K-12. Science 277:1453-1474

Bürgmann H, Howard EC, Ye W, Sun F, Sun S, Napierala S, Moran MA (2007) Transcriptional response of Silicibacter pomeroyi DSS-3 to dimethylsulfoniopropionate (DMSP). Environ Microbiol 9:2742-2755

Chou HT, Kwon DH, Hegazy M, Lu CD (2008) Transcriptome analysis of agmatine and putrescine catabolism in Pseudomonas aeruginosa PAO1. J Bacteriol 190: 1966-1975

Dasu VV, Nakada Y, Ohnishi-Kameyama M, Kimura K, Itoh Y (2006) Characterization and a role of Pseudomonas aeruginosa spermidine dehydrogenase in polyamine catabolism. Microbiology 152:2265-2272

DeLong EF, Preston CM, Mincer T, Rich V and others (2006) Community genomics among stratified microbial assemblages in the ocean's interior. Science 311:496-503

Giovannoni SJ, Rappe M (2000) Evolution, diversity, and molecular ecology of marine prokaryotes. In Kirchman DL (ed) Microbial ecology of the oceans. Wiley-Liss, Hoboken, NJ, p 47-84

Gonzalez JM, Covert JS, Whitman WB, Henriksen JR and others (2003) Silicibacter pomeroyi sp. nov. and Roseovarius nubinhibens sp. nov., dimethylsulfoniopropionatedemethylating bacteria from marine environments. Int $\mathrm{J}$ Syst Evol Microbiol 53:1261-1269

$>$ Higashibata H, Fujiwara S, Ezaki S, Takagi M, Fukui K, Imanaka T (2000) Effect of polyamines on histone-induced DNA compaction of hyperthermophilic archaea. J Biosci Bioeng 89:103-106

Höfle MG (1984) Degradation of putrescine and cadaverine in seawater cultures by marine bacteria. Appl Environ Microbiol 47:843-849

- Howard EC, Sun S, Biers EJ, Moran MA (2008) Abundant and diverse bacteria involved in DMSP degradation in marine surface waters. Environ Microbiol 10:2397-2410

Igarashi K, Kashiwagi K (1999) Polyamine transport in bacteria and yeast. Biochem J 344:633-642

Jorgensen NOG, Kroer N, Coffin RB, Yang XH, Lee C (1993) Dissolved free amino acids, combined amino acids and DNA as sources of carbon and nitrogen to marine bacteria. Mar Ecol Prog Ser 98:135-148

- Kroger N, Deutzmann R, Bergsdorf C, Sumper M (2000) Species-specific polyamines from diatoms control silica morphology. Proc Natl Acad Sci USA 97:14133-14138

Kumar S, Tamura K, Nei M (2004) MEGA3: integrated software for molecular evolutionary genetics analysis and sequence alignment. Brief Bioinform 5:150-163

Kurihara S, Oda S, Kato K, Kim HG, Koyanagi T, Kumagai H, Suzuki H (2005) A novel putrescine utilization pathway involves gamma-glutamylated intermediates of Escherichia coli K-12. J Biol Chem 280:4602-4608

Kusano T, Yamaguchi K, Berberich T, Takahashi Y (2007)

Editorial responsibility: Jed Fuhrman,

Los Angeles, California, USA
Advances in polyamine research in 2007. J Plant Res 120: $345-350$

Lee C, Jorgensen NOG (1995) Seasonal cycling of putrescine and amino acids in relation to biological production in a stratified coastal salt pond. Biogeochemistry 29:131-157

Lu CD, Itoh Y, Nakada Y, Jiang Y (2002) Functional analysis and regulation of the divergent spuABCDEFGH-spuI operons for polyamine uptake and utilization in Pseudomonas aeruginosa PAO1. J Bacteriol 184: 3765-3773

Marian FD, Garcia-Jimenez P, Robaina RR (2000) Polyamines in marine macroalgae: levels of putrescine, spermidine and spermine in the thalli and changes in their concentration during glycerol-induced cell growth in vitro. Physiol Plant 110:530-534

Moran MA, Buchan A, Gonzalez JM, Heidelberg JF and others (2004) Genome sequence of Silicibacter pomeroyi reveals adaptations to the marine environment. Nature 432:910-913

Mou X, Sun S, Edwards RA, Hodson RE, Moran MA (2008) Bacterial carbon processing by generalist species in the coastal ocean. Nature 451:708-711

Nishibori N, Yuasa A, Sakai M, Fujihara S, Nishio S (2001) Free polyamine concentrations in coastal seawater during phytoplankton bloom. Fish Sci 67:79-83

> Nishibori N, Matuyama Y, Uchida T, Moriyama T, Ogita Y, Oda M, Hirota H (2003) Spatial and temporal variations in free polyamine distributions in Uranouchi Inlet, Japan. Mar Chem 82:307-314

> Padmanabhan R, Kim K (1965) Oxidation of spermidine by a Pseudomonas sp. Biochem Biophys Res Commun 19:1-5

> Poretsky RS, Bano N, Buchan A, LeCleir G and others (2005) Analysis of microbial gene transcripts in environmental samples. Appl Environ Microbiol 71:4121-4126

> Rusch DB, Halpern AL, Sutton G, Heidelberg KB, Williamson S (2007) The Sorcerer II global ocean sampling expedition: Northwest Atlantic through Eastern Tropical Pacific. PLoS Biol 5:e77

> Saurin W, Dassa E (1994) Sequence relationships between integral inner membrane proteins of binding proteindependent transport systems: evolution by recurrent gene duplications. Protein Sci 3:325-344

Shaibe E, Metzer E, Halpern YS (1985) Metabolic pathway for the utilization of L-arginine, L-ornithine, agmatine, and putrescine as nitrogen sources in Escherichia coli K-12. J Bacteriol 163:933-937

Sowell SM, Wilhelm LJ, Norbeck AD, Lipton MS and others (2009) Transport functions dominate the SAR11 metaproteome at low-nutrient extremes in the Sargasso Sea. ISME J 3:93-105

Stover CK, Pham XQ, Erwin AL, Mizoguchi SD and others (2000) Complete genome sequence of Pseudomonas aeruginosa PAO1, an opportunistic pathogen. Nature 406: 959-964

Sumper M, Kroger N (2004) Silica formation in diatoms: the function of long-chain polyamines and silaffins. J Mater Chem 14:2059-2065

> Tabor CW, Tabor H (1985) Polyamines in microorganisms. Microbiol Rev 49:81-99

Tam R, Saier MH Jr (1993) Structural, functional, and evolutionary relationships among extracellular solute-binding receptors of bacteria. Microbiol Mol Biol Rev 57:320-346

Tyms AS (1989) Polyamines and the growth of bacteria and viruses. In: Bachrach U, and Heimer YM (ed) The physiology of polyamines, Vol II. CRS Press, Boca Raton, FL, p 3-33

Venter JC, Remington K, Heidelberg JF, Halpern AL and others (2004) Environmental genome shotgun sequencing of the Sargasso Sea. Science 304:66-74 